\title{
Disseminated BCG Infection (BCGosis) After BCG Vaccination
}

\author{
Poudel $\mathrm{P}^{1}$, Chitlangia $\mathrm{M}^{2}$
}

\begin{abstract}
BCG vaccine has excellent safety profile. Disseminated BCG infection, so called BCGosis and death have occurred in few cases, mainly in those with impaired immunity. We are reporting a seven month old infant who developed BCGosis after BCG vaccination. She presented with weight loss, fever, axillary lymphadenopathy and hepato-splenomegaly. She did not respond to standard antitubercular treatment. In the context of increasing number of reported cases of BCGois in HIV era, inoculation of BCG might be postponed in a suspected case of immune-deficiency disease, until it is ruled out.
\end{abstract}

\section{Introduction}

$\mathrm{B}$ CG (Bacilli Calmette-Guérin) vaccine is administered worldwide to prevent severe forms of tuberculosis and is recommended for routine use at birth in countries with high tuberculosis prevalence. It has an excellent safety profile with rare serious complications $^{1}$. Side effects- most commonly, ulceration at the vaccination site and regional lymphadenitis occur in $<1 \%$ to $10 \%$ of vaccinated individuals ${ }^{2}$. Disseminated BCG infection, so called BCGosis and death have occurred in 1 to 20 cases per 10 million doses administered, although this problem is restricted to persons with impaired immunity, such as children with severe combined immunodeficiency disease (SCID), chronic granulomatous disease (CGD) or with human immunodeficiency virus (HIV) infection ${ }^{2,3,4,5,6,7}$. We are reporting an infant admitted to BP Koirala Institute of Health Sciences, Dharan, Nepal, who had systemic BCG infection (BCGosis) after BCG vaccination.

\section{The Case}

A seven month age female baby presented to our hospital with history of progressively enlarging swelling at right axillary region with pus discharge for three months, fever for two months and progressive abdominal distension with weight loss for last one month. Child was vaccinated with BCG at 38 days of life. There was no history of contact to adult tuberculosis cases. Birth history was uneventful and development was optimal.

At the time of admission, the child was emaciated, pale and had an axillary temperature of $100.6^{\circ} \mathrm{F}$. The site of BCG vaccination
'Dr. Prakash Poudel, MBBS, MD, Associate Professor, ${ }^{2} \mathrm{Dr}$. Mohit Chitlangia, MBBS, MD, Senior Resident. Both from Department of Paediatrics and Adolescent Medicine, BP Koirala Institute of Health Sciences, Dharan, Nepal.

\section{Address for correspondence \\ Dr. Prakash Poudel \\ E-mail: prakashpdl@hotmail.com}

How to cite

Poudel P, Chitlangia M. Disseminated BCG Infection (BCGosis) After BCG Vaccination. J Nepal Paediatr Soc 2014;34(1):62-64.

doi: http://dx.doi.org/10.3126/jnps.v34i1.9679

This work is licensed under a Creative Commons Attribution 3.0 License.

(c) (i)

was indurated and erythematous. A soft to firm, mobile right axillary lymph node ( $4 \times 4 \mathrm{~cm}$ in size) was present which was discharging scanty amount of pus. On abdominal examination, there were firm hepatomegaly $(6 \mathrm{~cm}$ below subcostal margin) and firm splenomegaly ( $5 \mathrm{~cm}$ below subcostal margin).

Laboratory investigations revealed a leukocyte count of $21,300 \quad(35 \%$ lymphocytes) and hemoglobin of $7.5 \mathrm{~g} /$ dl. Erythrocyte sedimentation rate was $45 \mathrm{~mm}$ in $1^{\text {st }}$ hour. rK 39 test for visceral leishmaniasis and peripheral smear for malaria were negative. Ultrasound abdomen showed hepato-splenomegaly with multiple hypoechoic lesions within 
splenic parenchyma and mesenteric lymphadenopathy. Tuberculin test was non-reactive, microscopic examination of gastric aspirate was negative for acidfast bacilli and Gene Xpert (polymerase chain reaction test for $\mathrm{M}$ tuberculosis DNA) was also negative. Fine needle aspiration cytology from axillary lymph node showed the presence of acid fast bacilli. Antibody tests for HIV, Hepatitis B, Hepatitis C as and VDRL tests were negative.

When all investigations were completed, the child was already receiving two weeks of empirical antibiotic therapy but there was no response. We made diagnosis of disseminated BCG infection. Antitubercular treatment (ATT) with combination of four drugs (Isoniazid, Rifampin, Ethambutol and Pyrazinamide) was started. On re-assessment after one month of ATT, there was no improvement in any symptoms and signs. There were progressive enlargements of lymph node, liver and spleen. General condition was deteriorating and child was still febrile. Because of economic constraints and progressive deterioration in general condition despite treatment, parents denied further investigation and child was discharged home on ATT. There was no opportunity to evaluate the patient for primary immunodeficiency diseases.

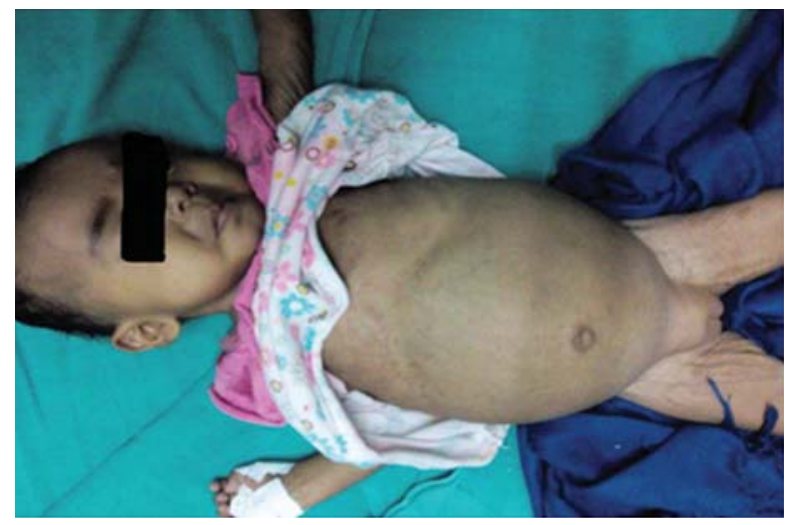

Fig 1: Infant with BCGosis showing severe wasting and abdominal distension due to hepatosplenomegaly

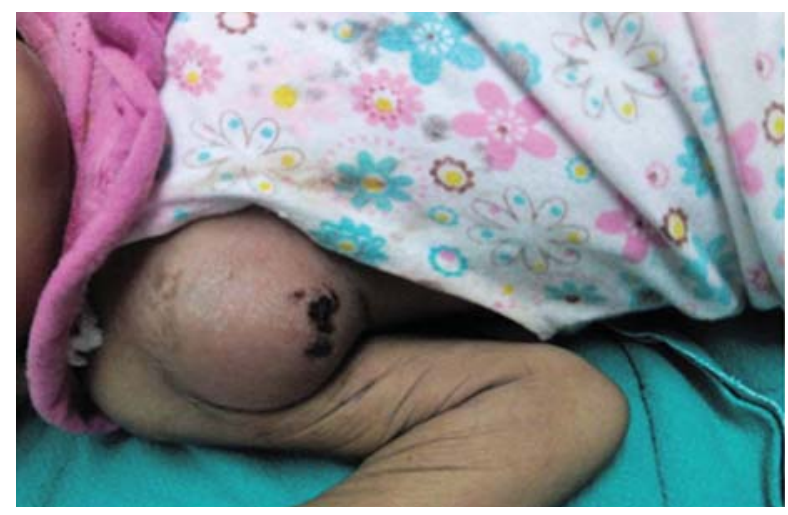

Fig 2: Showing axillary lymphadenitis near to BCG inoculation area in an infant with BCGosis

\section{Discussion}

The live attenuated Mycobacterium bovis-derived vaccine was first introduced in 1921. Since then billions of doses have been used and it is historically proven to be safe ${ }^{1}$. However, serious complications like disseminated BCG infection, also called BCGosis, rarely do occur. Disseminated BCG infection is found in 1 to nearly 20 cases per 10 million doses, given a mortality rate of $50 \%$ to $80 \%$, based on various studies ${ }^{2,8}$. Although serious complications of BCG vaccination including generalized lymphadenitis and disseminated infection do occur in immune-competentl hosts, immune-compromised infants and children are at greatest risk of developing BCGosis and most of the reported cases had, either acquired immunodeficiency like acquired immunodeficiency syndrome (AIDS) or primary immunodeficiency like SCID, CGD and other cell-mediated immune defects ${ }^{3,4,5,6,7,9}$. A rise in incidence is seen with higher rates in more recent studies, perhaps owing to AIDS $3,8,10,11$. Disseminated BCG disease has historically been a disease of infants and young children. Most of the reported cases are infants and young children. In a review study of 28 cases by Talbot et al., $82 \%$ of the cases occurred in children younger than 3 years old ${ }^{3}$. Disseminated BCG disease occurred in eight children younger than 6-months old and 12 patient younger than 12-months old out of 15 cases reported by Rezai et al $^{7}$. Our patient also was a 7-month-old female infant. But cases are now occurring also in adults and older children coinfected with human immunodeficiency virus ${ }^{3}$. Though exceptional, BCGosis have been reported in many immune-competent children also ${ }^{3,7}$. We ruled out the possibility of HIV infection but unfortunately, we did not have the opportunity and resources to evaluate our child for primary immunodeficiency.

In the review study of 28 cases by Talbot et al., it was shown that the most common site of dissemination, established by culture or histological demonstration of acid-fast bacilli, was lymph node, identified in $85 \%$ of cases. Blood and bone marrow were positive for BCG in one third of cases. Other common sites of dissemination were lungs, liver, spleen, skin and bone. The most commonly reported symptoms were fever, lymphadenopathy and weight loss. Failure to thrive and organomegaly were also common ${ }^{3}$. As we reported in our case, severe axillary lymphadenitis, hepatosplenomegaly and failure to thrive are common clinical features in many other reported cases ${ }^{6,9}$.

Our case did not respond to standard ATT. Response to therapy with standard ATT is generally reported to be 
poor in BCGosis cases with worst response in immunecompromised patients ${ }^{3,4,6}$. However, few cases were seen to respond to standard $\mathrm{ATT}^{3,4}$. More promising response have been seen when ATT is combined with interferon-gama in selected cases $^{7,12}$.

There are no statistics available in Nepal regarding incidence of BCGosis among vaccinated children. Infants in this country are routinely BCG-vaccinated, and there are many undiagnosed cases of primary or acquired immunodeficiency. Many of them are prone to develop this complication.

\section{Conclusion}

It is usually recommended to postpone inoculation of BCG for a few months in suspected cases of primary or acquired immunodeficiency disease, until appropriate screening tests exclude this diagnosis and vaccination should then be performed in those with an intact immune system. In the context of increasing number of HIV affected infants in Nepal, implementation of this recommendation in Nepal should be evaluated and considered.

\section{References}

1. Lugosi L. Theoretical and methodological aspects of BCG vaccine from the discovery of Calmette and Guerin to molecular biology. A review. Tuber Lung Dis 1992;73(5):252-61.

2. Raviglione MC, O’Brien RJ. Tuberculosis. In: Kasper DL, Braunwald E, Fauci AS, Hauser SL, Longo DL, Jameson JL, eds. Harrison's Principles of Internal Medicine. 16th ed. New York: McGraw-Hill; 2005. p.964.

3. Talbot EA, Perkins MD, Silva SF, Frothingham R. Disseminated bacille Calmette-Guerin disease after vaccination: case report and review. Clin Infect Dis 1997;24(6):1139-146.

4. Afshar Paiman S, Siadati A, Mamishi S, Tabatabaie P, Khotaee G. Disseminated Mycobacterium bovis infection after BCG vaccination. Iran J Allergy Asthma Immunol 2006;5(3):133-37.

5. Huang LH, Shyur SD, Weng JD, Shin-Chi, Tzen CY, Huang FY. Disseminated Bacille Calmette-Guérin disease as the initial presentation of X-linked severe combined immunodeficiency--a case report. Asian Pac J Allergy Immunol 2005;23(4):221-26.

6. Sadeghi-Shabestari M, Rezaei N. Disseminated bacille Calmette-Guérin in Iranian children with severe combined immunodeficiency. Int J Infect Dis 2009;13(6):e420-3.

7. Rezai MS, Khotaei G, Mamishi S, Kheirkhah M, Parvaneh N. Disseminated Bacillus CalmetteGuerin infection after BCG vaccination. J Trop Pediatr 2008;54(6):413-16.

8. Lotte A, Wasz-Hockert O, Poisson N, Engbaek $H$, Landmann $H$, Quast $U$ et al. Second IUATLD study on complications induced by intradermal BCGvaccination. Bull Int Union Tuberc Lung Dis 1988;63(2):47-59.

9. Santos A, Dias A, Cordeiro A, Cordinhã C, Lemos $S$, Rocha $G$ et al. Severe axillary lymphadenitis after BCG vaccination: alert for primary immunodeficiencies.. J Microbiol Immunol Infect 2010;43(6):530-37.

10. Edwards KM, Kernodle DS. Possible hazards of routine bacillus Calmette-Guerin immunization in human immunodeficiency virus-infected children. Pediatr Infect Dis J 1996;15(9):836-38.

11. Besnard M, Sauvion S, Offredo C, Gaudelus J, Gaillard JL, Veber F et al. Bacillus CalmetteGuerin infection after vaccination of human immunodeficiency virus-infected children. Pediatr Infect Dis J 1993;12(12):993-97.

12. Alangari AA, Al-Zamil F, Al-Mazrou A, Al-Muhsen $S$, Boisson-Dupuis S, Awadallah $S$ et al. Treatment of disseminated mycobacterial infection with high-dose IFN- $\gamma$ in patient. Clin Dev Immunol 2011;2011:691956. 